\title{
A fast and complete GEANT4 and ROOT Object-Oriented Toolkit: GROOT
}

\author{
D. Lattuada ${ }^{1, \star}$, D. L.Balabanski ${ }^{1}$, S. Chesnevskaya ${ }^{1}$, M. Costa ${ }^{2}$, V. Crucillà ${ }^{2}$, G. L. Guardo ${ }^{1}$, \\ M. La Cognata ${ }^{2}$, C. Matei ${ }^{1}$, R. G. Pizzone ${ }^{2}$, S. Romano ${ }^{2,3}$, C. Spitaleri ${ }^{2}, A$. Tumino ${ }^{2,4}$, and $Y . \mathrm{Xu}^{1}$ \\ ${ }^{1}$ ELI-NP / IFIN-HH, Magurele, Romania \\ ${ }^{2}$ INFN, Laboratori Nazionali del Sud, Catania, Italy \\ ${ }^{3}$ Dipartimento di Fisica e Astronomia, Università degli Studi, Catania, Italy \\ ${ }^{4}$ Facoltà di Ingegneria e Architettura, Università Kore, Enna, Italy
}

\begin{abstract}
Present and future gamma-beam facilities represent a great opportunity to validate and evaluate the cross-sections of many photonuclear reactions at near-threshold energies. Monte Carlo (MC) simulations are very important to evaluate the reaction rates and to maximize the detection efficiency but, unfortunately, they can be very cputime-consuming and in some cases very hard to reproduce, especially when exploring near-threshold cross-section. We developed a software that makes use of the validated tracking GEANT4 libraries and the n-body event generator of ROOT in order to provide a fast, realiable and complete MC tool to be used for nuclear physics experiments. This tool is indeed intended to be used for photonuclear reactions at $\gamma$-beam facilities with ELISSA (ELI Silicon Strip Array), a new detector array under development at the Extreme Light Infrastructure - Nuclear Physics (ELI-NP). We discuss the results of MC simulations performed to evaluate the effects of the electromagnetic induced background, of the straggling due to the target thickness and of the resolution of the silicon detectors.
\end{abstract}

\section{Introduction}

Nuclear reactions near threshold energies are of great interest in nuclear astrophysics. In the last few decades, direct and indirect methods have been shedding light on the uncertainties on nuclear crosssections at very low energies[1]. Nuclear physics experiments have a long history record and Monte Carlo (MC) simulations have always played a crucial role in their preparation, in data analysis and for proper understanding the experimental results. At ELI-NP[2] in Bucharest, together with the Laboratori Nazionali del Sud (LNS) of INFN in Catania, we are developing a MC tool to be used in nuclear physics experiments focused on complex detectors and nuclear reactions with low cross-sections, using open-source scientific libraries with a strong record of validation. The code was developed for being used in nuclear experiments with silicon detectors involving photonuclear reactions induced by the intense gamma beam produced by the Gamma Beam System (GBS) at ELI-NP. The idea of this toolkit initially came from the current lack of validated photonuclear models working at low energies in GEANT4[3] and the need of a GEANT4-based code to provide detailed information on electromagnetic (and hadronic) background in nuclear physics experiments. The open-source $\mathrm{C}++$ GROOT

\footnotetext{
^e-mail: dario.lattuada@eli-np.ro
} 


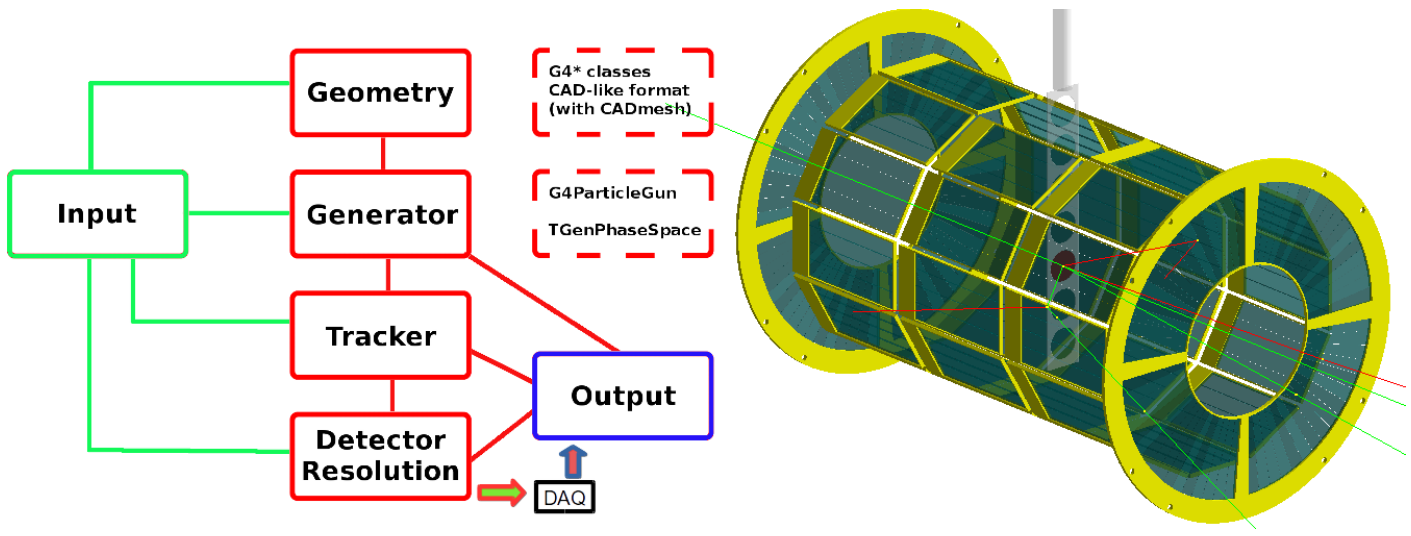

Figure 1. Left: A scheme of the MC code. Right: The geometry of ELISSA imported with CADMesh in GROOT (GEANT4 visualization interface). Gamma (green) and electron (red) tracks are shown.

(GEANT4 and ROOT Object-Oriented Toolkit) code we are currenlty developing mainly relies on ROOT[4], GEANT4 (10.02.p01) and the Standard Template Library (STL) of C++. Also, for the geometry we implemented the possibility to use CAD-like format as input, by means of CADMesh[5], a CAD Interface for GEANT4. The goal is to provide the users with a complete, realiable, fast and user-friendly framework to be used in the preparation, running and analysis phases of any nuclear physics experiments.

\section{The Code}

Since GROOT relies on GEANT4 for the most, it also follows its basic concepts and philosophy. The structure of the GROOT code (shown in Fig.1) follows a typical GEANT4 example. The program has both a classic event generator based on GEANT4 libraries and an external event generator (based on CERN ROOT libraries). Since photonuclear reactions near threshold are not properly handled by GEANT4 to date [6], the ROOT classes TGenPhaseSpace and TLorentzVector are used to provide a reliable and fast algorythm generating the ejectiles, starting from the input phase-space distribution. The geometry of the detector can be defined as a standard GEANT4 DetectorConstruction file by using G4 classes or imported in stl format by means of an external tool and its classes. The tracking of particles entirely relies on GEANT4 and its classes (default is G4EmStandardPhysics_option3). A (temporarily external) simple program is used to simulate the detector's angular and energy resolution. This will soon be incorporated into the main program. The output can be written to disk in ASCII, in ROOT format or both, with different levels of verbosity.

\section{Results}

The tool has been and will be used for past and future experiments. We present here some preliminary results of experiments with nuclear reactions of astrophysical interest with nearly monochromatic gamma beams with different profiles. We performed an experiment on ${ }^{7} \mathrm{Li}(\gamma, \mathrm{t})^{4} \mathrm{He}$ at $5-10 \mathrm{MeV}$ energies at $\mathrm{HI} \gamma \mathrm{S}$ (Duke University) in 2017 and this is a good candidate for zero-day experiment at ELINP. Also, the ${ }^{24} \operatorname{Mg}(\gamma, \alpha){ }^{20} \mathrm{Ne}$ reaction is an important reaction for the silicon burning in pre-supernova 


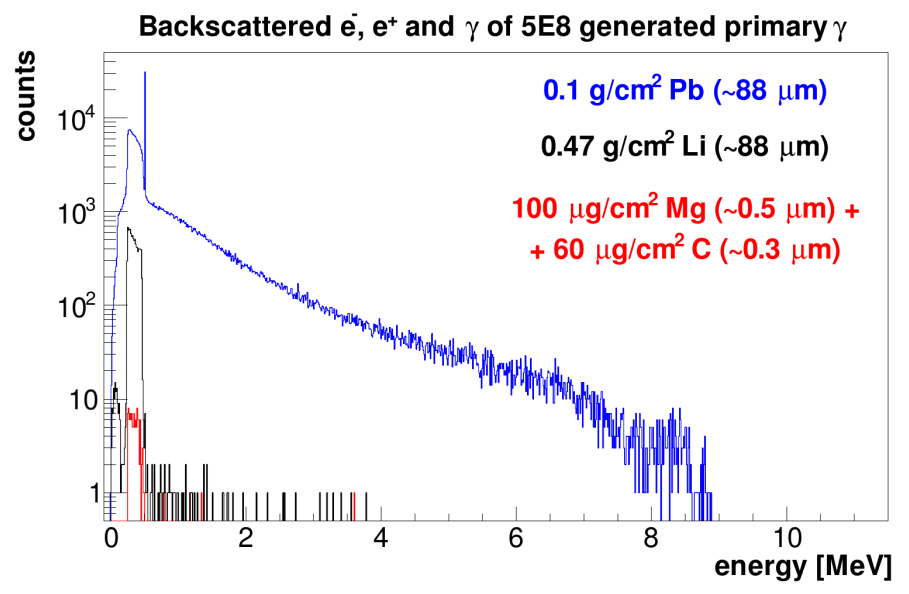

Figure 2. Backscattered electrons, positrons and gamma off a 11.4 MeV gamma beam wit ELI-NP GBS-like profile on different targets.

phase. Working with electromagnetic beams and silicon detectors the induced electromagnetic background can be an issue. We show the results of MC simulations with GROOT in Figure 2. Here we show the simulated expected background on ELISSA[7] due to a $11.4 \mathrm{MeV}$ gamma beam with ELINP profile $\left(2.5 \mathrm{~mm}\right.$ beam spot and $0.5 \%$ bandwith, $5 \mathrm{e}^{8}$ events are generated, corresponding just to few seconds of beam time) impinging on targets with different thickness and composition. Unsurprisingly enough, the rate of e.m. background (mainly made of $e^{-}$, but also $e^{+}$and $\gamma$ ) strongly depends on the target thickness and the atomic number of the element. It is clear that it is preferable to work with light elements and thin targets to avoid high rates that could negatively affect the threshold selection and damage your detectors eventually. For a realistic $250 \mu \mathrm{g} / \mathrm{cm}^{2}{ }^{24} \mathrm{Mg}$ target with a thin carbon backing, the expected e.m. background on ELISSA with the proposed GBS of ELI-NP is very low (10-100Hz, mostly at energies below the threshold). This does not fully apply for a thick target of lithium since the Compton scattering is significantly higher. Finally, the background is definitely huge for a thick lead target, as expected. Both the Compton scattering and the $e^{-} e^{+}$couple creation are important and they could represent an obstacle for proper particle detection, threshold selection, detector damage and also for the dead time.

We also show some simulation results for the $\mathrm{HI} \gamma \mathrm{S}$ experiment, where a 5-10MeV gamma beam with impinged on a lithium target to study the photodissociation of ${ }^{7} \mathrm{Li}(\gamma, \mathrm{t})^{4} \mathrm{He}$. In Figure 3 the energies and angles of tritons and alphas reaching the detector after losing energy inside the target are plotted on top (ELISSA setup is shown in Figure 1 for reference, only the X3 "rings" are considered). On bottom the same is plotted after applying a Gaussian smearing with an energy-dependent sigma in order to simulate the detector energy resolution, as reported from Micron and calculated in [8, 9]. The first proposed target was a $250 \mu \mathrm{g} / \mathrm{cm}^{2}$ lithium hydride while the second one was a slightly thinner gold-coated pure lithium target. The difference on the energy loss in the targets is evident. The choice of the target is thus strongly related to the energy spectra of the ejectiles. Estabilishing wether one is preferable over the other depends on the interplay between many factors, such as the precision of the measurement, the electronic noise and, last but not least, the target feasibility and robustness. Also, it should be noted that it might be usefeul to rotate the target in order to exploit the whole detector instead of dealing with a "blind" ring in the central ring at $90^{\circ}$ with respect to the beam direction. 


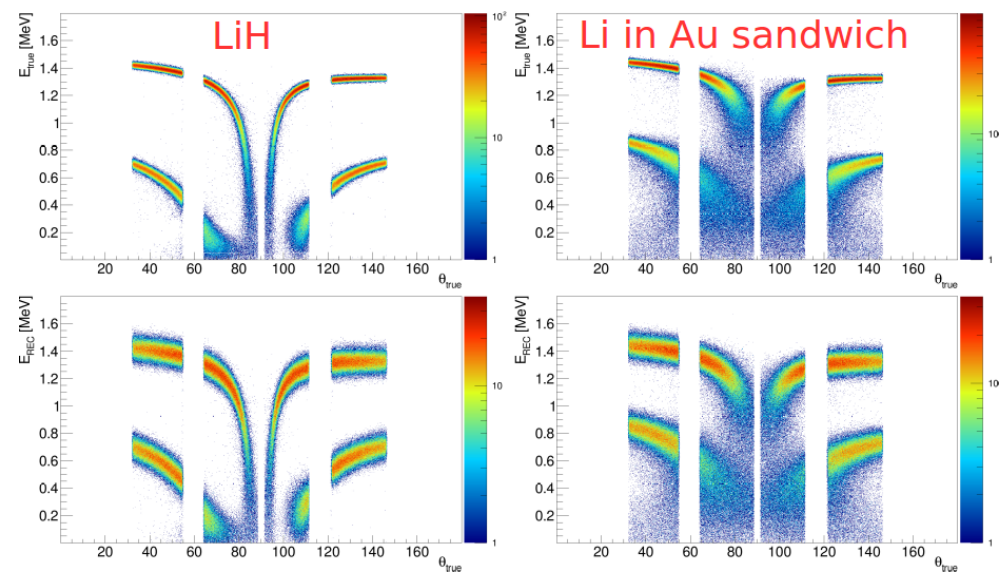

Figure 3. Top: For both LiH (left) and gold-coated lithium (right) targets, the energy of the simulated alpha particles from ${ }^{7} \mathrm{Li}(\gamma, \mathrm{t})$ reaction reaching the detector is plotted versus their angle of arrival. Bottom: the same but including the energy resolution effect.

In conclusion, even though a proper validation is going to be performed very soon, the reasonable results we show here are realiable since they are obtained with the use of widely validated libraries and classes from GEANT4 and ROOT. The code, still under development at ELI-NP and LNS, is applying to become the reference tool to be used for the photonuclear physics with silicon detectors at ELI-NP.

We acknowledge the support from the Extreme Light Infrastructure Nuclear Physics (ELI-NP) Phase II, a project cofinanced by the Romanian Government and the European Union through the European Regional Development Fund - the Competitiveness Operational Programme (1/07.07.2016, COP, ID 1334).

\section{References}

[1] O. Tesileanu et al., 2016 Charged particle detection at ELI-NP, Rom. Rep. Phys. 68 S699

[2] D. Filipescu et al., Eur. Phys. J., A51, 185 (2015)

[3] GEANT4: A Simulation toolkit - GEANT4 Collaboration (Agostinelli, S. et al.), Nucl. Inst. \& Meth. A506 (2003) 250-303 SLAC-PUB-9350, FERMILAB-PUB-03-339.

[4] ROOT - An Object Oriented Data Analysis Framework - Rene Brun and Fons Rademakers, Proceedings AIHENP'96 Workshop, Lausanne, Sep. 1996, Nucl. Inst. \& Meth. in Phys. Res. A 389 (1997) 81-86. See also http://root.cern.ch/.

[5] A CAD Interface for GEANT4, Poole, C. M. and Cornelius, I. and Trapp, J. V. and Langton, C. M., Australasian Physical \& Engineering Science in Medicine, September 2012,

[6] http://GEANT4.cern.ch/support/proc_mod_catalog/processes/hadronic/

G4PhotoNuclearProcess.html

[7] M. La Cognata et al., 2017 Journal of Instrumentation 12 C03079

[8] G. L. Guardo et al., this volume.

[9] S. Chesnevskaya et al., this volume. 